\title{
Capacitive Deionization for Selective Extraction of Lithium from Aqueous Solutions
}

\author{
M. Bryjak ${ }^{1, *}$, A. Siekierka ${ }^{1}$, J. Kujawski ${ }^{1}$, K. Smolińska-Kempisty ${ }^{1}$ and W. Kujawski ${ }^{2}$ \\ ${ }^{1}$ Department of Polymer and Carbon Materials, Wroclaw University of Technology, Wyb. Wyspianskiego 27, \\ 50-370 Wroclaw, Poland \\ ${ }^{2}$ Faculty of Chemistry, Nicolaus Copernicus University in Torun, 7 Gagarina str., 87-100 Torun, Poland
}

\begin{abstract}
The paper deals with extraction of lithium by means of two capacitive deionization systems: one composed of lithium selective electrode and second with electrode wrapped with Li-selective membrane. In the case of the first system, hybrid electrodes where obtained by mixing $\lambda-\mathrm{MnO}_{2}$ sorbent with activated carbon .The best Li-capacity was determined for electrode with $20 \mathrm{wt} . \mathrm{\%}$ of manganese oxide. For larger amounts of $\lambda-\mathrm{MnO}_{2}$ the electrode capacity decreased significantly. The second system was composed of carbon electrodes wrapped with ion-exchange membranes. The lithium selective membranes were synthesized by plasma induced interpolymerization of (meth)acrylic monomersinpores of Celgard 2400 support. Two functional monomers, poly(di(ethylene glycol)methyl ether methacrylate) and poly(glycidylmethacylate modified with hydroxymethyl-12-crown-4) were copolymerized with acrylic acid. It was found that the extraction of lithium chloride was the best for membrane caring copolymers of acrylic acid and glycidyl methacrylate modified with crown ether, andit was better than for membranes with sole poly(acrylic acid).
\end{abstract}

Keywords: Capacitive electrode, lithium selective membrane, lithium recovery, pore-filled membranes.

\section{INTRODUCTION}

Due to its robustness, energy efficiency and operation easiness, capacitive deionization (CDI) is being considered as an alternative method for water desalination [1-6]. The method is based on the use of electrostatic field between two porous electrodes and forcing the movements of ions to oppositely charged electrode. Ions are entrapped within the electrical double layer formed between bulk solution and electrode surface. When the electrical field is reversed, ions are released into the bulk solution and electrodes are regenerated to the next cycle. However, the majority of the CDI study was focused on water demineralization. The extraction of valuable ions from diluted solutions was of lower interest. Lately, few papers dealing with CDI-recovery of lithium from aqueous solutions have been published [7-9].

A search for new technologies for lithium recovery has gained the special interest lately due to growing demands for that element and limited its resources. When the usefulness of lithium-ion batteries for energy storage is well recognized $[10,11]$ the geographic distribution of lithium resources can cause some problems in the nearest future. It should be stressed that almost $80 \%$ of lithium it available in four countries only [12]. The largest amount of lithium appears in seawater, geothermal or brackish water and recovery

*Address correspondence to this author at the Department of Polymer and Carbon Materials, Wroclaw University of Technology, Wyb. Wyspianskiego 27, 50-370 Wroclaw, Poland; Tel: +4871 3202987;

E-mail: marek.bryjak@pwr.edu.pl of lithium from these sources appeals for specific technologies [13-15]. One of them is selective sorption that bases on intercalation of lithium ions into structure of inorganic sorbents, among them manganese oxide spinels are used mostly [16]. Unfortunately, the process of sorption is very slow and takes several weeks. What is more, the recovery of lithium needs application of strong acid that partially destroys the sorbent structure. The obtained acidic brine has to be neutralized with strong base that causes additional environmental problems.

The slow process of lithium adsorption on manganese oxide sorbents can be significantly accelerated by the use of electrical field. Ryu [7] has applied that approach to force cations to be absorbed by negatively charged lithium selective electrode. In the same time the second electrode, covered by anion exchange membrane, served to entrap the anions. The change of polarization caused desorption of ions and allowed to obtain concentrated solutions. The original paper of Ryu [7] did not mention about the use of any conducive additives in preparation of $\mathrm{MnO}_{2}$ electrodes. Hence, the elevated electrode resistance could be expected and, in consequence, a decrease of electrode capacity due to energy dissipation [2] could be predicted. Taking that into account, we tried to blend spinel of $\mathrm{MnO}_{2}$ with activated carbon to obtain more efficient electrodes. This approach is discussed in the Result section.

When ion-exchange membranes wrap CDI electrodes, a significant improvement in desalination is 
observed. Membranes allow counter-ions to be transported to electrode and block access co-ions. In the adsorption step counter-ions pass ion-exchange membranes and are entrapped in electrical double layer. In the desorption step ions are expelled from the double layer while their transfer to the second electrode is blocked by the ion-exchange membrane. Additionally, due membrane generated restriction of ion movements, the accumulation of co-ions takes place in the electrode macropores and its concentration exceeds ion concentration in the external solution. Hence, presence of membranes increases salt adsorption capacity of the system and the process is called membrane capacitive deionization, MCDI [2]. It is estimated that the use of ion-exchange membrane can duplicate the efficiency of salt removal [17]. In the case of use of selective ion-exchange membrane it is possible also to separate ions bearing the same sign. That phenomenon was observed for monovalent nitrate and chloride anions [18]. However, according to the best knowledge of the authors, there were no attempts to use selective membrane for separation of cations by means of the MCDI method. We have shown previously that it was possible to obtain membranes that can transport lithium ions preferentially $[19,20]$. Hence, such membranes can be used for wrapping the CDI cathode to generate selective transport of lithium cations. It is the second approach discussed in the Result section.

The goal of this paper was to compare two separation systems for capacitive extraction of lithium from aqueous solutions: one with Li-selective electrodes and second with Li-selective membranes. The comparison should point at the system that is worthy to be investigated deeply for harvesting lithium salts from diluted aqueous solutions.

\section{MATERIALS AND METHODS}

\section{Activated Carbon}

YP-50F Kuraray Chemical Co. steam activated coconut carbon was used throughout this study. Its characteristic can be found on the manufacturer web page [21].

\section{Manganese Oxide}

Spinel of $\mathrm{MnO}_{2}$ was prepared at Kitakyushu University according to procedure described in ref [22]. Before the use, sorbent was contacted with $1 \mathrm{M}$ aqueous solution of $\mathrm{HCl}$ for $12 \mathrm{hr}$.

\section{Poly(vinyl Chloride)}

PVC, Ongrovil S5070, was used as the binder. Its properties can be found on the manufacturer's specification available on the manufacturer webpage [23].

\section{Membrane and other Chemicals}

Celgard 2400 polypropylene membrane was used as the support. Acrylic acid (AA), glycidyl methacrylate (GLY), (di(ethylene glycol)methyl ether methacrylate (DEGMEM), ethylene glycol dimethacylate (EGDMA) were delivered by Aldrich.

\section{System}

To study the efficiency of lithium extraction in both approaches, a Fumatech laboratory electrodialyzer, FT-ED-100-4, was used. The stuck was composed of two carbon electrodes divided by a spacer of $200 \mu \mathrm{m}$ thickness. In all runs the anode was covered with Neosepta AMX membrane, while for evaluation of $\mathrm{Li}$ selectivity in MCDI process the selective membranes were fixed to cathode. In all runs $1.0 \mathrm{~V}$ was used. Lithium chloride solution, of total volume of $100 \mathrm{~mL}$, was circulated in the system with an average speed of $4 \mathrm{~L} / \mathrm{h}$. The electrosortion process was carried out for at least $24 \mathrm{hr}$. at room temperature.

\section{Preparation of Selective Electrode}

All electrodes were prepared by mixing $80 \%$ of activated carbon (or its blend with $\mathrm{MnO}_{2}$ ), $15 \%$ poly(vinyl chloride) and $5 \%$ of carbon black. Prior mixing the powdered activated carbon, or manganese oxide, was dried overnight at $105^{\circ} \mathrm{C}$. The cooled to room temperature powder was added to PVC solution in cyclohexanone with carbon black and stirred vigorously for $3 \mathrm{hr}$ with a mechanical stirrer. The slurry was cast on graphite current collector and electrode of $100 \mu \mathrm{m}$ thickness was formed by a casting knife. Solvent was allowed to evaporate at room temperature for two days and the residual amounts of it were removed in a vacuum dryer.

\section{Preparation of Lithium Selective Membrane}

\section{PAA/PEGMEM System}

Pore filled membranes with crosslinked acrylic acid/poly(di(ethylene glycol)methyl ether methacrylate) copolymer were prepared by plasma induced polymerization. For the purpose of this work mixture of 1:1 monomers were used. The procedure consisted of 
plasma activation of Celgard 2400 membrane, postreaction with oxygen and grafting of monomers to surface radicals activated by UV radiation. The membrane was placed into a dielectric barrier discharge reactor and treated with argon plasma. Both sides of the membrane were modified in the same way. After removal from the reactor, the samples were kept in air for $10 \mathrm{~min}$ and then they were immersed in $20 \mathrm{vol} \%$ aqueous solution of monomers. The solution was irradiated by UV radiation for $10 \mathrm{~min}$. To remove polymers not covalently bound to the polypropylene surface, samples were washed with large volume of water. The detailed procedure of membrane preparation was described previously [20]. In this preparation EGDMA was used in the 5 vol. \% amount with relation to other monomers.

\section{PAA/PGLY-Crown System}

Pore filled membranes with acrylic acid/poly (glycidylmethacylate modified with hydroxymethyl-12crown-4) were prepared similarly to procedure of PAA/PEGMEM membranes. However, to prevent opening oxirane rings the membrane grafting was carried out in mixture of liquid monomers ( $80 \mathrm{vol} . \%$ of glycidylmethactylate, $15 \mathrm{vol} \%$ of acrylic acid and 5 vol. \% of ethylene glycol dimethacrylate). The activated membranes were immersed in that mixture and kept in it for $3 \mathrm{hr}$ at room temperature. Then the membranes were surface dried, wrapped with poly(ethyl therephtalate) foil and placed in the drier at $60^{\circ} \mathrm{C}$. The grafting was carried out for $12 \mathrm{~h}$. The pore-filled membranes were rinsed with DI water and placed in $10 \%$ aqueous solution of 2-hydroxymethyl-12-crown-4. The modification was conducted overnight at $60^{\circ} \mathrm{C}$. The membranes were rinsed with large volume of DI water.

\section{Determination of Lithium Chloride Concentration}

Evaluation of $\mathrm{LiCl}$ concentration was carried out conductometrically. In some cases AAS analyses,
Analyst 100 Perkin-Elmer, were performed. In all experiments lithium chloride concentration was set to $10 \mathrm{mM}$.

\section{RESULTS}

\section{Lithium Selective Electrode}

Blending of manganese oxide with activated carbon was used to improve the electrode conductivity. The following contents of manganese oxide were selected for preparation of electrodes: $0 \%, 20 \%, 40 \%, 60 \%$ and 80 wt. \%. The obtained electrodes were tested in CDI mode and total adsorption of $\mathrm{LiCl}$ was calculated after $24 \mathrm{hr}$. of the process. The measured sorption capacity for evaluated electrodes is listed in Table 1.

The presented data fit well to the general trend in adsorption capacity described by Suss [24] where regular CDI system is able to uptake up $15 \mathrm{mg}$ of salt per one gram of carbon electrode while composite system (carbon electrode and metal oxide) takes up to $20 \mathrm{mg}$ per gram. In the case of spinel studied in this paper, its addition up to $20 \%$ was profitable but additional portion of the manganese oxide destroyed the electrode capacity. It was anticipated that two effects caused that phenomenon: a rapid drop of electrode conductivity caused by manganese oxide and redox reactions. When the first effect was certain the second one needed an additional explanation. We noted some brownish spots on the electrode surface that could indicate the oxidation processes. It was plausible that manganese reduced from +4 to +2 of its oxidation state while chloride anions oxidized to chlorine oxide ions that destroyed the components of the electrodes: activated carbon and/or polymer binder. Some indirect proofs of this process can be found in the paper of Ryu [8] where lithium hydroxide was used. It means the use of lithium chloride caused some problems in the process. In consequence we decided to not continue studies on preparation of lithium

Table 1: Salt Adsorption Capacity of the CDI System Composed of Activated Carbon Anode and $\mathrm{MnO}_{2} \mathrm{Modified}$ Cathode

\begin{tabular}{|c|c|c|}
\hline $\begin{array}{c}\mathbf{M n O}_{2} \text { content in cathode } \\
{[\%]}\end{array}$ & $\begin{array}{c}\text { Salt adsortion capacity } \\
\text { [mg LiCl/g electrode] }\end{array}$ & Remarks \\
\hline \hline 0 & 12.2 & Smooth process \\
\hline 20 & 16.7 & Smooth process \\
\hline 40 & 6.0 & Conductivity slight instable \\
\hline 60 & 4.7 & Sudden changes of conductivity, brownish spots on the electrode surface \\
\hline 80 & 0.48 & Sudden changes of conductivity, brownish spots on the electrode surface \\
\hline
\end{tabular}


selective electrodes by blending manganese oxide and activated carbon. It is expected that preparation of stable CDI electrodes is possible by blending activated carbon with other lithium selective adsorbents.

\section{Lithium Selective Membranes - DEGMEM Case}

Our previous studies have shown that pore-filled membranes with copolymer of poly(acrylic acid-codi(ethyleneglycol methyl)ether methacrylate), P(AA-coDEGMEM), crosslinked with EGDMA were able to transport lithium more efficiently than membranes grafted with poly(acrylic acid) only [20]. What was more we noted that lithium transport process at elevated $\mathrm{pH}$ values was more facilitated than transfer of other ions. To check if these membranes could be applied for MCDI separation we evaluated the kinetics of electrosorption. The obtained relations are shown in Figure 1.

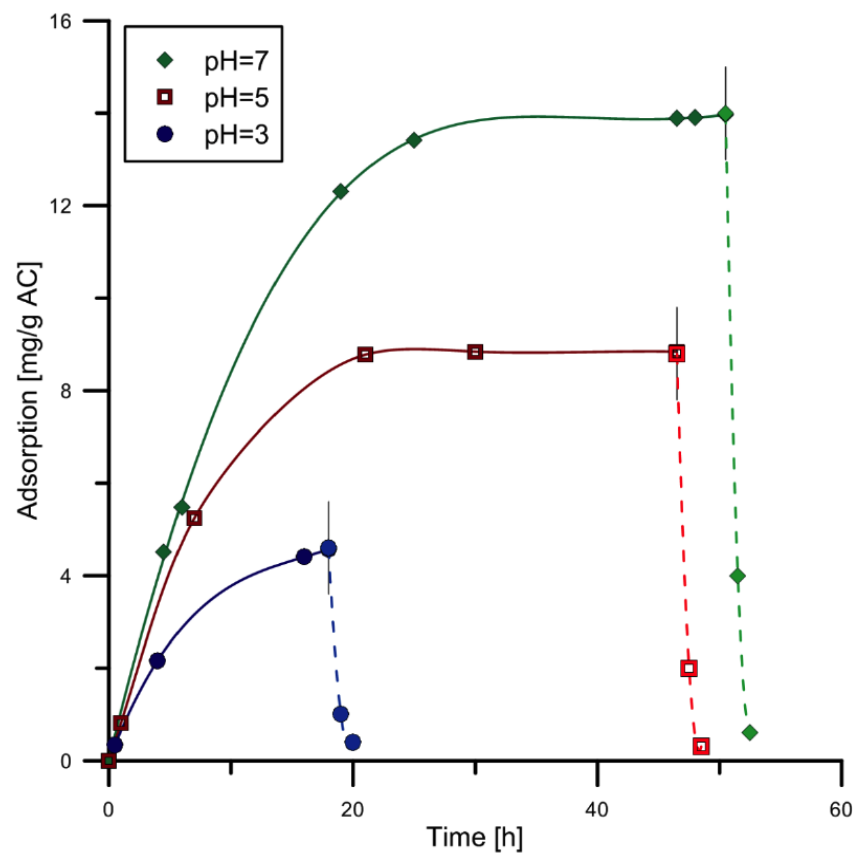

Figure 1: Kinetics of $\mathrm{LiCl}$ adsorption in MCDI process with $\mathrm{P}(\mathrm{AA}$-co-DEGMEM) membrane. Effect of solution $\mathrm{pH}$. The broken lines - electrodesorption curves.

It is seen that system was able to adsorb more lithium chloride when carboxylic groups were in ionic form. Similar effect was observed in dialytic system studied previously [19]. On that base we concluded that rearrangement of polymer chains and formation of conductive tracks with $-\mathrm{CH}_{2}-\mathrm{CH}_{2}$-O- segments was a reason of lithium transport improvement. Hence, the described membranes can be used for lithium separation as most of natural sources of lithium have neutral or alkaline $\mathrm{pH}$ values.
Evaluation of flux improvement, FI, for potassium, sodium and lithium chloride transport allowed to compared separation efficiency of membranes with both pore filled structures: crosslinked poly(acrylic acid) and crosslinked copolymer P(AA-co-DEGMEM).The effect of solution $\mathrm{pH}$ on flux improvement is shown in Table 2 [25].

Flux improvement was calculated as

$\mathrm{FI}=\left(\mathrm{J}_{\mathrm{PAA}-\mathrm{CO}-\mathrm{DEGMEM}}-\mathrm{J}_{\text {PAA }}\right) / \mathrm{J}_{\text {PAA }}$

where JPAA-CO-DEGMEM and $J_{\text {PAA }}$ are fluxes through membranes with $\mathrm{P}(\mathrm{AA}-\mathrm{Co}-\mathrm{DEGMEM})$ and $\mathrm{PAA}$, respectively.

Table 2: Salt Flux Improvement

\begin{tabular}{|c|c|c|}
\hline \multirow{2}{*}{} & \multicolumn{2}{|c|}{ PAA-DEGMEM } \\
\cline { 2 - 3 } & $\mathbf{p H = 5 . 5}$ & $\mathbf{p H = 3 . 0}$ \\
\hline \hline $\mathrm{KCl}$ & $67 \%$ & 0 \\
\hline $\mathrm{NaCl}$ & $40 \%$ & 0 \\
\hline $\mathrm{LiCl}$ & $90 \%$ & 0 \\
\hline
\end{tabular}

It was noted that the flux of lithium chloride was almost duplicated when membrane contained DEGMEM units and when process was carried out in solutions with $\mathrm{pH}$ above dissociation constant of carboxylic groups. That improvement was not so evident for other alkaline chlorides.

\section{Lithium Selective Membranes - PGLY-Crown ether Case}

Here two-step synthesis route was applied. Monomer of glycidyl methacrylate was polymerized within the pores and the obtained polymer was modified with hydroxymethyl-12-crown-4. The plasma activated samples of Celgard membrane were swollen in the reaction mixture composed of $A A, G L Y$ and EGDMA. The swollen membranes were wrapped with poly(ethyl therephtalate) foils and polymerized at elevated temperature. Finally, the obtained membranes were modified with hydroxymethyl-12-crown-4.

The average increase of membrane weight for each step was as follows: PAA-128 $\div 133 \%$, PAA-coGLY $190 \div 192 \%$ and PAA-Co-GLY-crown ether $195 \div 199 \%$. It meant some amounts of glycidyl methacrylate were grafted and its reactive oxirane groups were able to bind hydroxymethyl 12-crown-4 ether. 
The prepared AA-GLY-crown ether membranes were tested in the MCDI system in extraction of $\mathrm{LiCl}$. To evaluate the process selectivity extraction of $\mathrm{KCl}$ was conducted also.

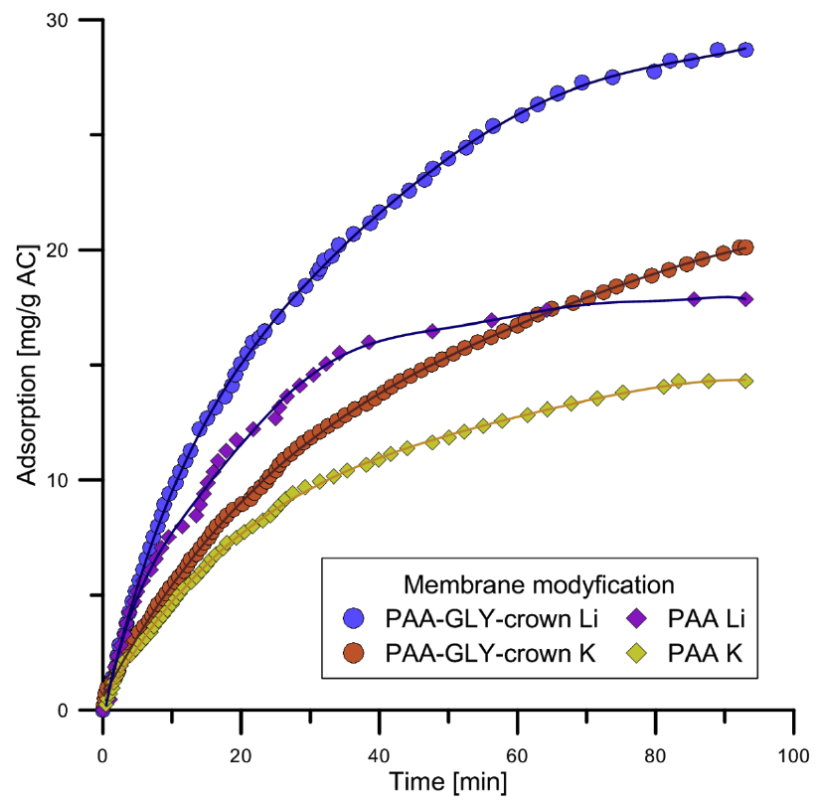

Figure 2: $\mathrm{LiCl}$ and $\mathrm{KCl}$ adsorption in MCDI system with PAA and PAA-GLY-crown membranes. Solution $\mathrm{pH} 5.5$, concentration of both chlorides $10 \mathrm{mM}$.

It is observed similar relationship as for AADEGMEM case: copolymer of PAA-GLY-crown was more efficient in transportation of lithium than PAA. It meant both kind of functional groups could interact in transportation of lithium. What is more, the comparison of adsorption capacity showed that the evaluated system could adsorb about $30 \mathrm{mg} \mathrm{LiCl}$ per 1 gram of activated carbon. It is three times more than uptake determined in the PAA-DEGMEM system. Hence, the membranes with 12-crown-4 ether ligands are more efficient in transportation of lithium than diethylene glycol groups.

To check if PAA-GLY-crown retained its selectivity, the process of lithium sorption/desorption was repeated few times. Electrosorption kinetics for three cycles is shown in Figure 3.

The studies showed that membrane did not change its ability for separation of lithium with repeated number of cycles. This finding is in line with Omosebi's conclusion [26] that long term electrosortion capacity of MCDI does not change.

\section{CONCLUSIONS}

The studies have shown that the use of manganese oxide for formation of selective electrodes was not so

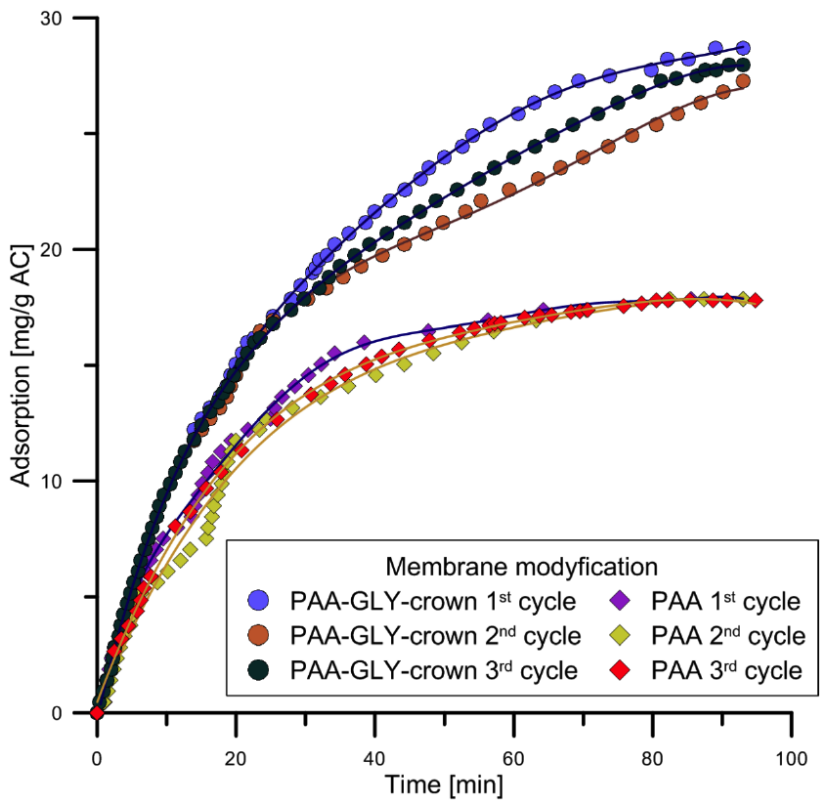

Figure 3: Consecutive adsorption kinetics for PAA-GLYcrown (round) and PAA (diamond) membranes. For clarity of the picture three runs were presented only.

profitable as expected. Electrodes prepared by blending manganese oxide with activated carbon adsorbed less lithium than electrodes without $\mathrm{MnO}_{2}$. The presence of manganese oxide accelerated formation of aggressive chlorine oxides that deteriorated the electrode surface. MCDI method with Li-selective membranes, facilitated the extraction process. Among two types of evaluated membranes, these bearing the crown- 4 ether ligands were more efficient. What is more, the presence of carboxylic groups in the membranes improved the sorption capacity of the system. The use of MCDI configuration allowed to extract much more lithium salt than the regular CDI arrangement and separation process was stable in several repetition. Hence, the more perspective method for lithium recovery seems to be MCDI with lithium selective membranes.

\section{ACKNOWLEDGEMENT}

The work was supported by Wroclaw Research Centre EIT+ within the project "The Application of Nanotechnology in Advanced Materials" - NanoMat (POIG.01.01.02-02-002/08) co-financed by the European Regional Development Fund (Operational Program of Innovative Economy, 1.1.2.)

\section{REFERENCE}

[1] Liu Y, Nie Ch, Liu X, Xu X, Sun Z, Pan L. Review on carbonbased composite materials for capacitive deionization. RSC Adv 2015; 5: 15205-15225.

http://dx.doi.org/10.1039/C4RA14447C 
[2] Porada S, Zhao R, van der Wal A, Presser V, Biesheuvel PM. Review on the science and technology of water, desalination by capacitive deionization. Prog Mater Sci 2013; 58: $1388-1442$.

http://dx.doi.org/10.1016/j.pmatsci.2013.03.005

[3] Porada S, Weinstein L, Dash R, van der Wal A, Bryjak M, Gogotsi Y, Biesheuvel PM. Water desalination using capacitive deionization with microporous carbon electrodes. ACS Appl Mater Interfaces 2012; 4: 1194-1199. http://dx.doi.org/10.1021/am201683

[4] Porada S, Borchardt L, Oschatz M, Bryjak M, Atchison JS, Keesman KJ, Kaskel S, Biesheuvel PM, Presser V. Direct prediction of the desalination performance of porous carbon electrodes for capacitive deionization. Energy Environ Sci 2013; 6: 3700-3712.

http://dx.doi.org/10.1039/c3ee42209g

[5] Anderson MA, Cudero AL, Palma J. Capacitive deionization as an electrochemical means of saving energy and delivering clean water. Comparison to present desalination practices: Will it compete? Electrochim Acta 2010; 55: 3845-3856. http://dx.doi.org/10.1016/j.electacta.2010.02.012

[6] Oren Y. Capacitive deionization (CDI) for desalination and water treatment - past, present and future (a review). Desalination 2008; 228: 10-29.

http://dx.doi.org/10.1016/j.desal.2007.08.005

[7] Ryu T, Lee DH, Ryu JC, Shin J, Chung KS, Kim YH. Lithium recovery system using electrostatic field assistance. Hydrometallurgy 2015; 151: 78-83.

http://dx.doi.org/10.1016/j.hydromet.2014.11.005

[8] Ryu T, Ryu JC, Shin J, Lee DH, Kim YH, Chung KS. Recovery of lithium by an electrostatic field-assisted desorption process. Ind Eng Chem Res 2013; 52: 1373813742. http://dx.doi.org/10.1021/ie401977s

[9] Kim S, Lee J, Kang JS, Jo K, Kim S, Sung YE, Yoon J. Lithium recovery from brine using a $\mathrm{MnO}_{2}$ /activated carbon hybrid supercapacitor system. Chemosphere 2015; 125: 5056.

http://dx.doi.org/10.1016/j.chemosphere.2015.01.024

[10] Prior T, Wager PA, Stamp A, Widmer R, Giurco D. Sustainable governance of scarce metals: The case of lithium. Sci Total Environ 2013; 461-462: 785-791. http://dx.doi.org/10.1016/j.scitotenv.2013.05.042

[11] Gruber PW, Medina PA, Keoleian GA, Kesler SE, Everson MP, Wallington TJ. Global lithium availability: A constraint for electric vehicles? J Ind Ecol 2011; 15: 760-774. http://dx.doi.org/10.1111/j.1530-9290.2011.00359.x

[12] Vikstrom H, Davidsson S, Hook M. Lithium availability and future production outlooks. Appl Energy 2013; 110: 252-266. http://dx.doi.org/10.1016/.apenergy.2013.04.005

[13] Ma LW, Chen BZ, Chen Y, Shi XC. Preparation, characterization and adsorptive properties of foam-type lithium adsorbent. Micropor Mesopor Mater 2011; 142: 147153.

http://dx.doi.org/10.1016/j.micromeso.2010.11.028
[14] Miyai $\mathrm{Y}$, Ooi K, Katoh S. Recovery of lithium from seawater using a new type of ion-sieve adsorbent based on $\mathrm{MgMn}_{2} \mathrm{O}_{4}$. Sep Sci Technol 1988; 23: 179-191. http://dx.doi.org/10.1080/01496398808057641

[15] Wang L, Ma W, Liu R, Li HY, Meng CG. Correlation between $\mathrm{Li}+$ adsorptioncapacity and the preparation conditions of spinel lithium manganese precursor. Solid State lonics 2006; 177: $1421-1428$.

http://dx.doi.org/10.1016/j.ssi.2006.07.019

[16] Park J, Sato H, Nishihama S, Yoshizuka K. Lithium recovery from geothermal water by combined adsorption methods. Solvent Extr Ion Exch 2012; 30: 398-404. http://dx.doi.org/10.1080/07366299.2012.687165

[17] Kim JS, Kim CS, Shin HS, Rhim JW. Application of synthesized anion and cation exchange polymers to membrane capacitive deionization (MCDI). Macromol Res 2015; 23: 360-366. http://dx.doi.org/10.1007/s13233-015-3049-6

[18] Kim YJ, Choi JH. Selective removal of nitrate ion using a novel composite carbon electrode in capacitive deionization. Water Res 2012; 46: 6033-6039.

http://dx.doi.org/10.1016/j.watres.2012.08.031

[19] Smolińska-Kempisty K, Bryjak M, Wolska J, Kujawski W. pHsensitive membranes for lithium separation. Mater Chem Phys 2014; 148: 548-553. http://dx.doi.org/10.1016/j.matchemphys.2014.08.003

[20] Smolińska-Kempisty K, Bryjak M. Stimuli response polypropylene membranes as selective separators for alkaline ions. Desalination 2012; 300: 64-69. http://dx.doi.org/10.1016/j.desal.2012.06.006

[21] Kuraray Chemical Co., Web page: Available from: http://www.kuraraychemical.com/products/sc/capacitor.htm

[22] Park J, Sato H, Nishihama S, Yoshizuka K. Separation and recovery of lithium from geothermal water by sequential adsorption process with $\mathrm{MnO}_{2}$ and $\mathrm{TiO}_{2}$. Ion Exch Lett 2012; 5: $1-5$.

[23] Borsod Chem LTD. Web page, Available from: http://www.borsodchem-pvc.comdefault.aspx

[24] Suss ME, Porada S, Sun X, Biesheuvel PM, Yoon J, Presser V. Water desalination via capacitive deionization: what is it and what can we expect from it? Energy Environ Sci 2015; 8: 2296-2319.

http://dx.doi.org/10.1039/C5EE00519A

[25] Bryjak M, Smolinska-Kempisty K, Kujawski J. Separation of lithium by membrane enhanced capacitive deionization, Proceedings of Intl Conference on lon Exchange, Okinawa 912 November 2014.

[26] Omosebi A, Gao X, Landon J, Liu K. Asymmetric electrode configuration for enhanced membrane capacitive deionization. ACS Appl Mater Interfaces 2014; 6: 1264012649.

http://dx.doi.org/10.1021/am5026209 